International Institute for Applied Systems Analysis - A-2361 Laxenburg - Austria Tel: +432236807 • Fax: +43223671313 • E-mail: info@iiasa.ac.at •Web: www.iiasa.ac.at

INTERIM REPORT IR-98-038/April

\title{
Adaptive dynamics in Allele space: Evolution of Genetic Polymorphism by Small Mutations in a Heterogeneous Environment
}

É.Kisdi (kisdi@ludens.elte.hu)

S.A.H. Geritz(sgeritz@mailhost.utu.fi)

Approved by

Ulf Dieckmann (dieckman@iiasa.ac.at)

Project Coordinator, Adaptive Dynamics Network

Interim Reports on work of the International Institute for Applied Systems Analysis receive only limited review. Views or opinions expressed herein do not necessarily represent those of the Institute, its National Member Organizations, or other organizations supporting the work. 


\section{IIASA STUdies In AdAPTIVE Dynamics No. 25}

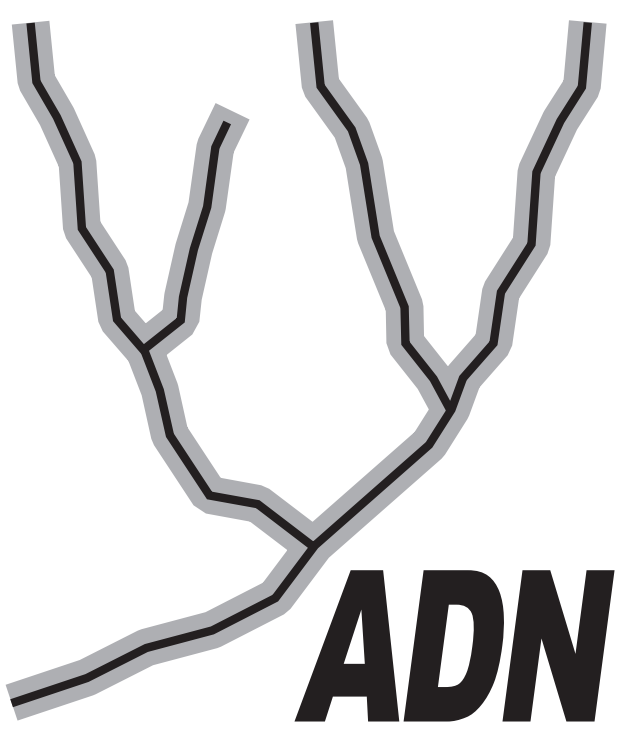

The Adaptive Dynamics Network at IIASA fosters the development of new mathematical and conceptual techniques for understanding the evolution of complex adaptive systems.

Focusing on these long-term implications of adaptive processes in systems of limited growth, the Adaptive Dynamics Network brings together scientists and institutions from around the world with IIASA acting as the central node.

Scientific progress within the network is reported in the IIASA Studies in Adaptive Dynamics series.

\section{The Adaptive Dynamics Network}

The pivotal role of evolutionary theory in life sciences derives from its capability to provide causal explanations for phenomena that are highly improbable in the physicochemical sense. Yet, until recently, many facts in biology could not be accounted for in the light of evolution. Just as physicists for a long time ignored the presence of chaos, these phenomena were basically not perceived by biologists.

Two examples illustrate this assertion. Although Darwin's publication of "The Origin of Species" sparked off the whole evolutionary revolution, oddly enough, the population genetic framework underlying the modern synthesis holds no clues to speciation events. A second illustration is the more recently appreciated issue of jump increases in biological complexity that result from the aggregation of individuals into mutualistic wholes.

These and many more problems possess a common source: the interactions of individuals are bound to change the environments these individuals live in. By closing the feedback loop in the evolutionary explanation, a new mathematical theory of the evolution of complex adaptive systems arises. It is this general theoretical option that lies at the core of the emerging field of adaptive dynamics. In consequence a major promise of adaptive dynamics studies is to elucidate the long-term effects of the interactions between ecological and evolutionary processes.

A commitment to interfacing the theory with empirical applications is necessary both for validation and for management problems. For example, empirical evidence indicates that to control pests and diseases or to achieve sustainable harvesting of renewable resources evolutionary deliberation is already crucial on the time scale of two decades.

The Adaptive Dynamics Network has as its primary objective the development of mathematical tools for the analysis of adaptive systems inside and outside the biological realm. 


\section{IIASA StUdies in AdAPTIVe Dynamics}

No. 1 Metz JAJ, Geritz SAH, Meszéna G, Jacobs FJA, van Heerwaarden JS: Adaptive Dynamics: A Geometrical Study of the Consequences of Nearly Faithful Reproduction.

IIASA Working Paper WP-95-099.

van Strien SJ, Verduyn Lunel SM (eds.): Stochastic and Spatial Structures of Dynamical Systems, Proceedings of the Royal Dutch Academy of Science (KNAW Verhandelingen), North Holland, Amsterdam, pp. 183-231 (1996).

No. 2 Dieckmann U, Law R:

The Dynamical Theory of Coevolution: A Derivation from Stochastic Ecological Processes.

IIASA Working Paper WP-96-001.

Journal of Mathematical Biology (1996) 34, 579-612.

No. 3 Dieckmann U, Marrow P, Law R:

Evolutionary Cycling of Predator-Prey Interactions: Population Dynamics and the Red Queen.

Journal of Theoretical Biology (1995) 176, 91-102.

No. 4 Marrow P, Dieckmann U, Law R:

Evolutionary Dynamics of Predator-Prey Systems: An Ecological Perspective.

IIASA Working Paper WP-96-002.

Journal of Mathematical Biology (1996) 34, 556-578.

No. 5 Law R, Marrow P, Dieckmann U:

On Evolution under Asymmetric Competition.

IIASA Working Paper WP-96-003.

Evolutionary Ecology (1997) 11, 485-501.

No. 6 Metz JAJ, Mylius SD, Diekmann O:

When Does Evolution Optimise? On the Relation between Types of Density Dependence and Evolutionarily Stable Life History Parameters.

IIASA Working Paper WP-96-004.

No. 7 Ferrière R, Gatto M:

Lyapunov Exponents and the Mathematics of Invasion in Oscillatory or Chaotic Populations.

Theoretical Population Biology (1995) 48, 126-171.

No. 8 Ferrière R, Fox GA:

Chaos and Evolution.

Trends in Ecology and Evolution (1995) 10, 480-485.

No. 9 Ferrière R, Michod RE:

The Evolution of Cooperation in Spatially Heterogeneous Populations.

IIASA Working Paper WP-96-029.

American Naturalist (1996) 147, 692-717. 
No. 10 Van Dooren TJM, Metz JAJ:

Delayed Maturation in Temporally Structured Populations with Non-

Equilibrium Dynamics.

IIASA Working Paper WP-96-070.

Journal of Evolutionary Biology (1998) 11, 41-62.

No. 11 Geritz SAH, Metz JAJ, Kisdi E, Meszéna G:

The Dynamics of Adaptation and Evolutionary Branching.

IIASA Working Paper WP-96-077.

Physical Review Letters (1997) 78, 2024-2027.

No. 12 Geritz SAH, Kisdi E, Meszéna G, Metz JAJ:

Evolutionarily Singular Strategies and the Adaptive Growth and Branching of the Evolutionary Tree.

IIASA Working Paper WP-96-114.

Evolutionary Ecology (1998) 12, 35-57.

No. 13 Heino M, Metz JAJ, Kaitala V:

Evolution of Mixed Maturation Strategies in Semelparous Life-Histories:

the Crucial Role of Dimensionality of Feedback Environment.

IIASA Working Paper WP-96-126.

Philosophical Transactions of the Royal Society of London Series B (1997) 352, $1647-1655$.

No. 14 Dieckmann U:

Can Adaptive Dynamics Invade?

IIASA Working Paper WP-96-152.

Trends in Ecology and Evolution (1997) 12, 128-131.

No. 15 Meszéna G, Czibula I, Geritz SAH:

Adaptive Dynamics in a Two-Patch Environment: a Simple Model for Allopatric and Parapatric Speciation.

IIASA Interim Report IR-97-001.

Journal of Biological Systems (1997) 5, 265-284.

No. 16 Heino M, Metz JAJ, Kaitala V:

The Enigma of Frequency-Dependent Selection.

IIASA Interim Report IR-97-061.

Trends in Ecology and Evolution (1998) in press.

No. 17 Heino M:

Management of Evolving Fish Stocks.

IIASA Interim Report IR-97-062.

Canadian Journal of Fisheries and Aquatic Sciences (1998) in press.

No. 18 Heino M:

Evolution of Mixed Reproductive Strategies in Simple Life-History Models.

IIASA Interim Report IR-97-063.

No. 19 Geritz SAH, van der Meijden E, Metz JAJ:

Evolutionary Dynamics of Seed Size and Seedling Competitive Ability.

IIASA Interim Report IR-97-071.

No. 20 Galis F, Metz JAJ:

Why are there so many Cichlid Species? On the Interplay of Speciation and Adaptive Radiation.

IIASA Interim Report IR-97-072.

Trends in Ecology and Evolution (1998) 13, 1-2. 
No. 21 Boerlijst MC, Nowak MA, Sigmund K:

Equal Pay for all Prisoners. / The Logic of Contrition.

IIASA Interim Report IR-97-073.

AMS Monthly (1997) 104, 303-307.

Journal of Theoretical Biology (1997) 185, 281-294.

No. 22 Law R, Dieckmann U:

Symbiosis without Mutualism and the Merger of Lineages in Evolution.

IIASA Interim Report IR-97-074.

Proceedings of the Royal Society of London Series B (1998) 265, 1245-1253.

No. 23 Klinkhamer PGL, de Jong TJ, Metz JAJ:

Sex and Size in Cosexual Plants.

IIASA Interim Report IR-97-078.

Trends in Ecology and Evolution (1997) 12, 260-265.

No. 24 Fontana W, Schuster P:

Shaping Space: The Possible and the Attainable in RNA Genotype-

Phenotype Mapping.

IIASA Interim Report IR-98-004.

No. 25 Kisdi E, Geritz SAH:

Adaptive Dynamics in Allele Space: Evolution of Genetic Polymorphism by Small Mutations in a Heterogeneous Environment.

IIASA Interim Report IR-98-038.

No. 26 Fontana W, Schuster P:

Continuity in Evolution: On the Nature of Transitions.

IIASA Interim Report IR-98-039.

Science (1998) 280, 1451-1455.

No. 27 Nowak MA, Sigmund K:

Evolution of Indirect Reciprocity by Image Scoring. / The Dynamics of

Indirect Reciprocity.

IIASA Interim Report IR-98-040.

Nature (1998) 393, 573-577.

No. 28 Kisdi E:

Evolutionary Branching Under Asymmetric Competition.

IIASA Interim Report IR-98-045.

Issues of the IIASA Studies in Adaptive Dynamics series can be obtained free of charge. Please contact:

Adaptive Dynamics Network

International Institute for Applied Systems Analysis

Schloßplatz 1

A-2361 Laxenburg

Austria

Telephone +432236 807, Telefax +43 2236 71313, E-Mail adn@iiasa.ac.at, Internet http://www.iiasa.ac.at/Research/ADN 


\section{Contents}

Introduction 1

The Model 4

Adaptive Dynamics 5

Pairwise Invasibility Plots 5

$\begin{array}{lr}\text { Polymorphic Singularities } & 10\end{array}$

$\begin{array}{ll}\text { Bifurcation Analysis } & 13\end{array}$

$\begin{array}{ll}\text { Monomorphic singularities } & 13\end{array}$

$\begin{array}{ll}\text { Polymorphic singularities } & 14\end{array}$

$\begin{array}{ll}\text { Evolution with multiple attractors } & 17\end{array}$

$\begin{array}{ll}\text { Discussion } & 21\end{array}$

Evolution of genetic polymorphism in heterogeneous environments 21

$\begin{array}{ll}\text { Adaptive dynamics in diploid populations } & 24\end{array}$

$\begin{array}{ll}\text { References } & 27\end{array}$

Appendix A 32

Simulated evolutionary trees $\quad 32$

Appendix B 34

$\begin{array}{ll}\text { Unique equilibrium allele frequencies in two-allele polymorphisms } & 34\end{array}$

Appendix C 36

$\begin{array}{ll}\text { Monomorphic singularities } & 36\end{array}$ 


\begin{abstract}
We investigate how a genetic polymorphism of distinctly different alleles can evolve in an initially monomorphic population under frequency-dependent selection if mutations have only a small phenotypic effect. We consider the case of a single additive locus with a continuum of potential allele types in a diploid outbreeding population. As a specific example, we use a version of Levene's (1953) soft selection model, where stabilizing selection acts on a continuous trait within each of two habitats. If the optimal phenotypes within the habitats are sufficiantly different, then two distinctly different alleles evolve gradually from a single ancestral allele. In a wide range of parameter values, the two locally optimal phenotypes will be realized by one of the homozygotes and the heterozygote, rather than the two homozygotes. Unlike in the haploid analogue of the model, there can be multiple polymorphic evolutionary attractors with different probabilities of convergence.
\end{abstract}

Keywords: adaptive dynamics, ESS, evolutionary branching, frequency dependent selection, genetic polymorphism, mutation-limited evolution, soft selection model 


\author{
About the Authors \\ Éva Kisdi \\ Department of Genetics, Eötvös University \\ 1088 Budapest, Múzeum krt. 4/A, Hungary \\ and \\ Department of Zoology, University of Maryland, \\ College Park, MD 20742, USA \\ Stefan A.H. Geritz \\ Department of Zoology, University of Maryland \\ College Park, MD 20742, USA
}

\title{
Address for correspondence:
}

Until 31 July, 1998:

Éva Kisdi, Adaptive Dynamics Network, International Institute for Applied Systems Analysis; e-mail: kisdi@iiasa.ac.at.

After 15 August, 1998:

Éva Kisdi, Department of Mathematics, University of Turku, FIN-20500 Turku, Finland, Fax: 358-21-633-6595; e-mail any time: kisdi@ludens.elte.hu. 


\section{Acknowledgments}

We are grateful to Géza Meszéna and Hans Metz for many discussions on adaptive dynamics. Peter Abrams helped us with valuable comments on an earlier version of the manuscript. A discussion with Joseph Hofbauer led us to the proof in Appendix B. Ulf Dieckmann and Andrea Pugliese kindly shared their unpublished work. A substantial part of this research was done while É.K. received a scholarship from Collegium Budapest, Institute for Advanced Study in Budapest, Hungary, and enjoyed the wonderful working conditions provided by this institute. This work was supported by the Hungarian Science Foundation, OTKA (T 019272). 


\section{Adaptive Dynamics in Allele Space: Evolution of Genetic Polymorphism by Small Mutations in a Heterogeneous Environment}

É. Kisdi

S.A.H. Geritz

\section{Introduction}

Frequency dependent selection arises under a wide variety of ecological situations such as multiple-niche environments, resource competition, predation, etc. There are two approaches frequently used in theoretical analyses of frequency dependent evolution of continuous traits. The first searches for evolutionarily stable strategies (Maynard Smith, 1982), or evolutionarily stable coalitions of coexisting strategies (Brown and Vincent, 1987a,b, 1992; Brown and Pavlovic, 1992) as the possible final states of evolution. The second models the dynamics of how traits change in time, most often by difference equations describing directional evolution (e.g., Hofbauer and Sigmund, 1990; Marrow et al., 1992, 1996; Abrams et al., 1993a; Vincent et al., 1993; Matsuda and Abrams, 1994a,b; Dieckmann et al., 1995; Dieckmann and Law, 1996; Abrams et Matsuda, 1997; Law et al., 1997); these models are largely compatible with quantitative genetic models (Charlesworth, 1990; Iwasa et al., 1991; Taper and Case, 1992; Abrams et al., 1993a,b; Taylor, 1996). 
The attractors of directional evolution need not be evolutionarily stable strategies (Eshel, 1983; Taylor, 1989; Nowak, 1990; Christiansen, 1991; Abrams et al., 1993a; Vincent et al., 1993; Eshel et al., 1997). Metz et al. (1996) and Geritz et al. $(1997,1998)$ show that the attractors which lack local evolutionary stability are evolutionary branching points, at which two coexisting strategies evolve from a single ancestral strategy by small mutational steps (similar results were obtained independently by Eshel et al., 1997).

The evolution of polymorphism at a branching point proceeds as follows. As long as the monomorphic population is away from the branching point, a mutant somewhat nearer the branching point can invade and replace the resident. When directional evolution has arrived at the neighbourhood of a branching point, the invading mutant does not replace the former resident anymore, but the two phenotypically similar strategies form a protected dimorphism. Once dimorphic, the population undergoes disruptive evolution during which the two strategies grow further and further apart. The emergence of two widely distinct strategies by small evolutionary steps in an initially monomorphic population can be envisaged as branching on the evolutionary tree.

Protected polymorphism and disruptive selection near a branching point have been indicated in a number of specific models (Christiansen and Loeschcke, 1980; Van Tienderen and De Jong, 1986; Christiansen, 1991; Brown and Pavlovic, 1992; Abrams et al. 1993a). Specific examples of evolutionary branching have been investigated by Metz et al. (1992, 1996), Doebeli (1996a), Doebeli and Ruxton (1997), Meszéna et al. (1997), Geritz et al. (1998), Doebeli and Dieckmann (in press), Geritz and Kisdi (in press), Mathias and Kisdi (in press), Meszéna and Metz (in press), Geritz et al. (in prep.). The evolutionary attractors corresponding to fitness minima found, for example, by Cohen and Levin (1991), Ludwig and Levin (1991), Vincent et al. (1993), Doebeli (1996b), and Law et al. (1997) are evolutionary branching points. 
An important limitation of the adaptive dynamics framework used for modelling evolutionary branching (Metz et al., 1996; Geritz et al., 1997, 1998) is the assumption of haploid or clonal inheritance. In this paper we investigate how this framework can be applied to diploid sexually reproducing organisms. Since in diploid populations alleles (rather than phenotypes) undergo mutation and are passed from one generation to the next, we model evolution in allele space by keeping track of the full allelic composition of the population. Evolutionary branching in allele space produces genetic polymorphism of alleles with distinctly different phenotypic effects via a series of small mutations. Specifically, we address the following questions:

(1) Does genetic polymorphism evolve by evolutionary branching under similar ecological conditions where phenotypic adaptive dynamics leads to branching?

(2) What are the qualitative differences between evolution in haploid and diploid populations?

In order to tackle these questions, we use a continuous version of Levene's soft selection model (Levene, 1953) as an example, because of its relative simplicity and because of its well-known population genetics (see, for example, Roughgarden, 1979; Maynard Smith, 1989). In the original model of Levene (1953), there is one locus with a fixed number of pre-defined alleles all of which are already present in the population. Here we assume an initially monomorphic population, and a continuum of potential allele types that could arise by mutation. Mutations are assumed to have small but finite (i.e., not infinitesimally small) phenotypic effect. We investigate (1) under which conditions polymorphism evolves via evolutionary branching, (2) the existence of evolutionarily stable polymorphisms, and (3) how the number and properties of evolutionary attractors change under different patterns of environmental heterogeneity. Finally we show that if there are multiple polymorphic 
evolutionary attractors, any of them may be reached by the population, but the probability of evolving to one or another may be markedly different.

\section{The Model}

Consider a population of an annual organism in an environment consisting of two habitats or patches. Within each patch, there is first a period of selection, followed by nonselective competition. During selection, the probability of survival is a Gaussian function of a continuous phenotypic trait, $x$. The patches have different optimal phenotypes, $m_{1}$ and $m_{2}$, respectively, but have the same width of the fitness function, $\sigma$. The survival probability of an individual with phenotype $x$ is thus

$$
\begin{aligned}
& f_{1}(x)=\alpha_{1} \exp \left(-\frac{\left(x-m_{1}\right)^{2}}{2 \sigma^{2}}\right) \text { in patch 1, and } \\
& f_{2}(x)=\alpha_{2} \exp \left(-\frac{\left(x-m_{2}\right)^{2}}{2 \sigma^{2}}\right) \text { in patch 2, }
\end{aligned}
$$

where $\alpha_{1}, \alpha_{2}$ are arbitrary constants of proportionality. Without loss of generality, we assume that $m_{1}=-d / 2$ and $m_{2}=d / 2$, where $d$ is the difference between the optimal phenotypes.

During the competition phase, a fixed number of adults survive within each patch, such that a fraction $c_{1}$ of the adult population is recruited from the first patch, and the remaining fraction $c_{2}=1-c_{1}$ from the second patch ("soft selection"; Levene, 1953). Offspring are produced by random mating in the entire population, and are distributed over the patches randomly.

The trait under selection, $x$, is determined by a single locus with a continuum of possible alleles. Each allele is represented by the phenotype of the corresponding homozygote. In order to avoid excessive notation, we shall use the same symbol, e.g. $x$, to denote an allele and to denote the phenotype of an individual 
homozygous for this allele. The alleles act additively on the phenotype, the phenotype of a heterozygote being exactly half-way between the homozygotes. Notice that this does not imply additive fitnesses, since the fitness functions (Eqs. 1) are nonlinear.

The alleles may undergo mutation. In the analysis of the model, we assume that mutations are of small (but not infinitesimally small) phenotypic effect. Evolution is mutation-limited, i.e., mutations occur infrequently such that a mutant allele either has been excluded or has spread, and the population has reached its equilibrium, by the time the next mutant comes along. To test the robustness of the model predictions, we performed direct simulations of the evolutionary process, in which we relaxed the assumption of strict mutation limitation (see Appendix A for the simulation methods).

\section{Adaptive Dynamics}

\section{Pairwise Invasibility Plots}

Consider a rare mutant allele, $y$, in a resident population which is otherwise monomorphic for allele $x$. The rare allele is present almost exclusively in heterozygotes with phenotype $(x+y) / 2$. During the trait-dependent selection phase, the frequency of the mutant allele increases by a factor $f_{i}\left(\frac{x+y}{2}\right) / f_{i}(x)$ within patch $i(i=1$ or 2$)$. The marginal fitness of $y$ in the entire population is

$$
W_{x}(y)=c_{1} \frac{f_{1}\left(\frac{x+y}{2}\right)}{f_{1}(x)}+c_{2} \frac{f_{2}\left(\frac{x+y}{2}\right)}{f_{2}(x)}
$$

(cf. Roughgarden, 1979, p. 232). If $W_{x}(y)$ is greater than one, the mutant allele can spread, otherwise it is excluded from the population. 
A convenient way to summarize which mutant allele can invade a monomorphic resident population is to construct a so-called pairwise invasibility plot, which indicates for each pair of resident and mutant alleles whether $W_{x}(y)$ is greater or smaller than 1 (Van Tienderen and De Jong, 1986; Metz et al., 1992, 1996; Kisdi and Meszéna, 1993, 1995; Geritz et al., 1997, 1998). In Fig. 1 (top panels), the dotted areas are those resident-mutant pairs where the mutant can invade; in the clear areas the mutant dies out. Since the resident allele neither spreads nor vanishes (so that $W_{x}(x)=1$ ), the main diagonal $(y=x)$ is one of the border lines between the 'invasion' $\left(W_{x}(y)>1\right)$ and 'noninvasion' $\left(W_{x}(y)<1\right)$ areas.

If a mutant allele invades, it may either replace the original resident, or it may form a polymorphism with the original allele. A pair of alleles forms a protected polymorphism if both of them can spread when rare, i.e., if both $W_{x}(y)$ and $W_{y}(x)$ are greater than one. These pairs can be visualized by taking the mirror image of the pairwise invasibility plot along its main diagonal, and superimposing the mirror image on the original: The area where the 'invasion' parts of the mirror image and the original overlap defines the possible protected polymorphisms (Fig. 1, bottom panels). All two-allele polymorphisms in this model are protected (see Appendix B).

If mutations are of small phenotypic effect $(|y-x| \leq \delta 1)$, a mutant cannot form a polymorphism with the resident unless the population is in the neighbourhood of $x^{*}$, the so-called evolutionarily singular allele (Fig. 1). As long as the population is away from $x^{*}$, invasion implies allele substitution, and recurrent allele substitutions result in directional evolution. In both examples shown in Fig. 1, directional evolution proceeds towards the singular allele $x^{*}$. 


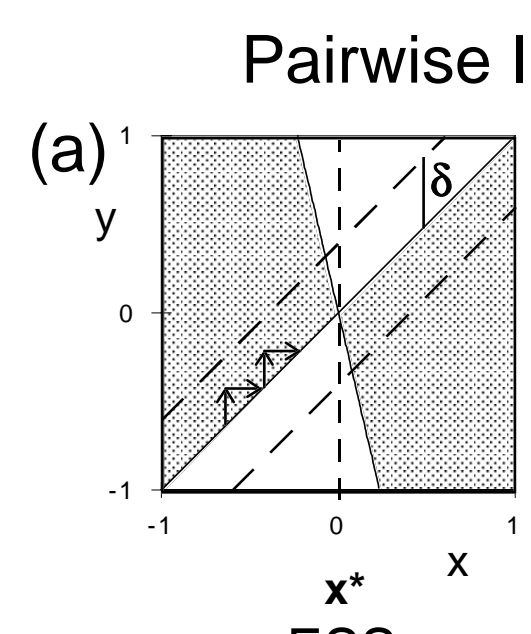

ESS

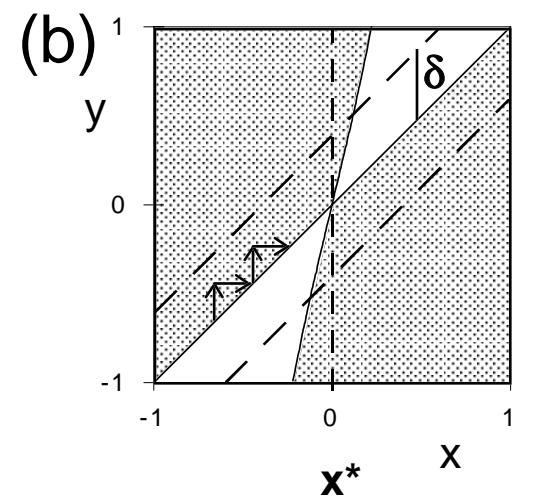

branching point

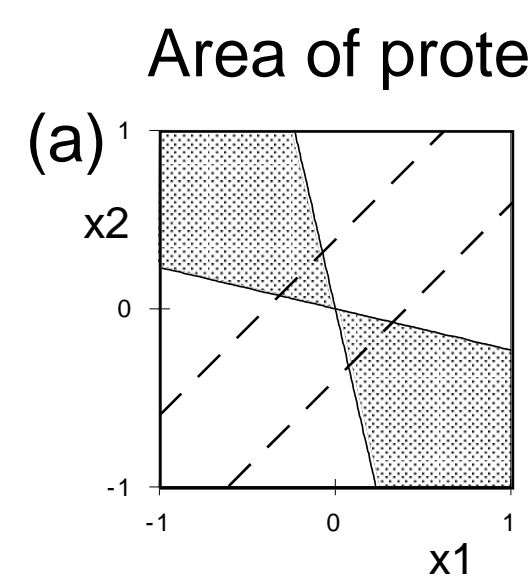

ected polymorphism

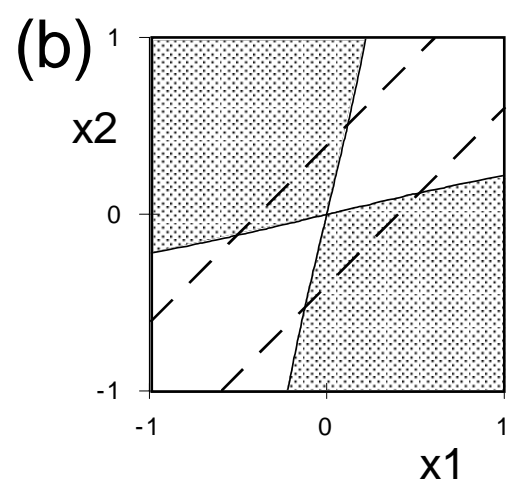

Figure 1. Pairwise invasibility plots (top) and the area of protected polymorphism (bottom) for two parameter sets. Inside the dotted areas of the pairwise invasibility plots the mutant allele $(y)$ can invade the resident $(x)$, whereas in the clear areas the mutant dies out. The arrows indicate the replacement of the original resident by its mutant. The region between the dashed lines along the diagonal is feasible by small mutations $(|y-x| \leq \delta 2)$. In the bottom panels, dotted areas denote the allele pairs $\left(x_{1}, x_{2}\right)$ which form a protected polymorphism. (a) $d / \sigma=1, c_{1}=0.5 ; x^{*}=0$ is a convergence stable ESS. (b) $d / \sigma=3, c_{1}=0.5 ; x^{*}=0$ is convergence stable but evolutionarily unstable, i.e., it is a branching point.

Geritz et al. (1998) provide a full classification of possible types of evolutionary singularities together with the dynamical characteristics of evolution taking place nearby. For the present purposes we shall consider only two properties of the singularities: (i) convergence stability, i.e., whether the singularity is an attractor 
of evolution proceeding by a series of allele replacements (Eshel, 1983; Taylor, 1989; Christiansen, 1991), and (ii) local evolutionary stability, i.e., whether a population monomorphic for the singular allele is resistant to invasions by nearby mutants.

The top panel of Fig. 1a shows a pairwise invasibility plot when the two patches are similar in their respective optimal phenotypes $\left(d / \sigma=1, c_{1}=0.5\right) . x=0$ is an evolutionarily stable allele, since there is no mutant which could invade a population that is monomorphic for $x^{*}$. Notice that in the neighbourhood of $x^{*}$ there are pairs of alleles which can form protected polymorphisms (Fig. 1a, bottom panel), so that a population evolving towards $x^{*}$ may become polymorphic before it reaches the singularity. This polymorphism, however, can be invaded by alleles still nearer to $x^{*}$, and therefore will eventually be resolved at the evolutionarily stable allele (Eshel et al., 1997; Geritz et al., 1998).

If the optimal phenotypes within the two patches are more different, the convergence stable singularity lacks evolutionary stability, i.e., it is a branching point. In Fig. $1 \mathrm{~b}\left(d / \sigma=3, c_{1}=0.5\right)$, a population that is monomorphic for $x^{*}=0$ can be invaded by both smaller and larger mutants, and the mutant allele forms a protected polymorphism with the original resident. The population therefore necessarily becomes polymorphic in the neighbourhood of $x^{*}$. In this polymorphic population only mutants outside the two resident alleles can invade, and invasion is followed by the elimination of the allele in the middle. Repeated invasions of mutants thus give rise to a series of polymorphisms of two increasingly distinct alleles (Eshel et al., 1997; Geritz et al., 1998). Simulated evolutionary trees with branching are shown in Fig. 2. 
(a)
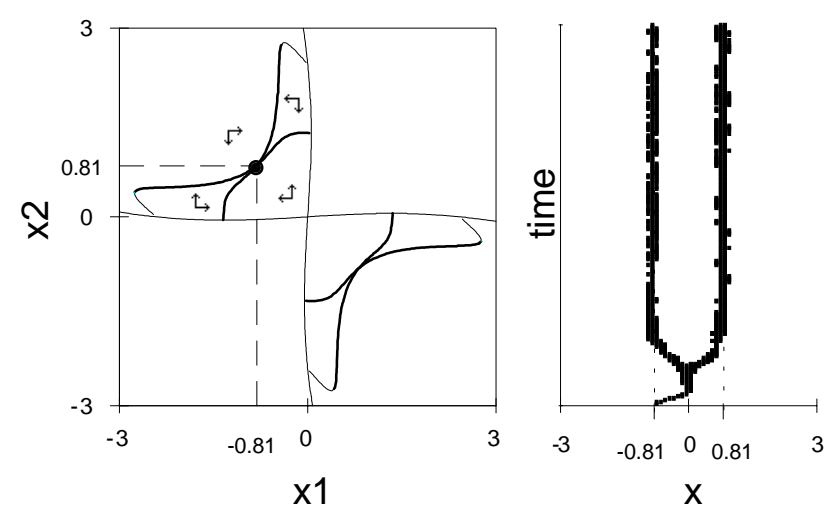

(b)
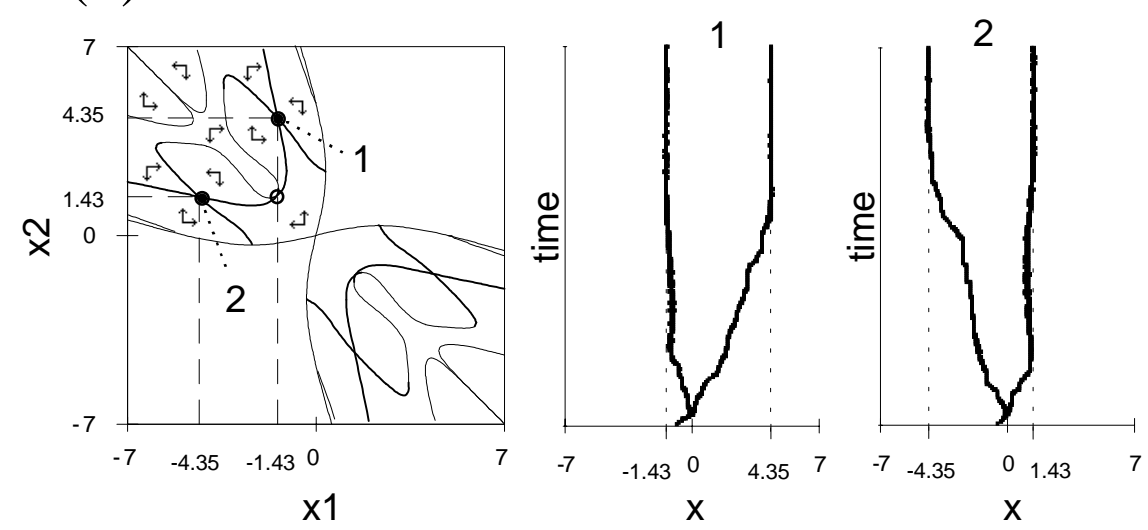

(c)
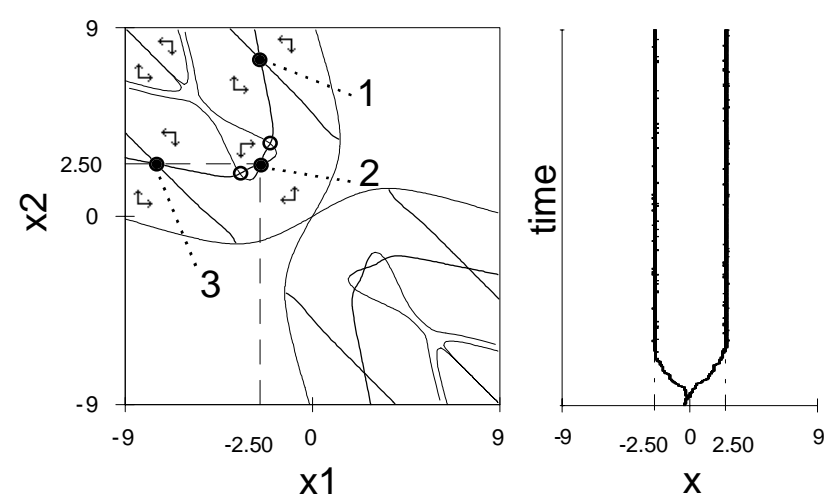

Fig. 2. Isocline plots and corresponding simulated evolutionary trees. In the isocline plots, horizontal (vertical) arrows indicate in which direction the allele $x_{1}$ $\left(x_{2}\right)$ can evolve. At the $x_{1^{-}}\left(x_{2}-\right)$ isoclines, the direction of the horizontal (vertical) arrows changes. Thick isoclines are evolutionarily stable, thin isoclines are evolutionarily unstable. Filled circles denote convergence stable polymorphisms, open circles are saddle points. The evolutionary trees show the alleles present in direct simulations (time span of 300000 generations). $c_{1}=0.5$; (a) $d / \sigma=2.25,(b)$ $d / \sigma=3,(c) d / \sigma=5$. 


\section{Polymorphic Singularities}

In order to model evolution after an evolutionary branching has taken place, we need to generalize the analysis for polymorphic populations. Consider a population with two distinctly different resident alleles, $x_{1}$ and $x_{2}$, in population genetical equilibrium. Analogously to Eq. 2, the marginal fitness of a rare mutant allele, $y$, is

$$
W_{x_{1}, x_{2}}(y)=c_{1} \frac{p f_{1}\left(\frac{y+x_{1}}{2}\right)+q f_{1}\left(\frac{y+x_{2}}{2}\right)}{\left\langle f_{1}\right\rangle}+c_{2} \frac{p f_{2}\left(\frac{y+x_{1}}{2}\right)+q f_{2}\left(\frac{y+x_{2}}{2}\right)}{\left\langle f_{2}\right\rangle}
$$

where $p$ and $q=1-p$ are the equilibrium allele frequencies of $x_{1}$ and $x_{2}$, respectively, and $\left\langle f_{i}\right\rangle=p^{2} f_{i}\left(x_{1}\right)+2 p q f_{i}\left(\frac{x_{1}+x_{2}}{2}\right)+q^{2} f_{i}\left(x_{2}\right)$ is the average survival probability during trait-dependent selection in patch $i$.

Evolution can be described solely in terms of alleles present only if the marginal fitness of a mutant allele is unequivocally determined by the types of the resident alleles, $x_{1}$ and $x_{2}$. In polymorphic populations this requires that there is a unique stable equilibrium of allele frequencies, $p$ and $q$, for each possible pair of resident alleles. The diploid two-allele, two-patch Levene model with arbitrary genotypic fitnesses may have up to three polymorphic population genetical equilibria, two of which can be stable. In Appendix B we derive sufficient conditions under which there is at most one polymorphic equilibrium, and show that this condition is always fulfilled if the within-patch fitness is a Gaussian function of an additively determined phenotypic trait. In the present version of the Levene model, therefore, any complication arising from multiple population genetical equilibria need not be considered. Models of adaptive dynamics with several attractors of the resident population were considered by Rand et al. (1994), Doebeli and Ruxton (1997), and Geritz et al. (in prep.). 
For each pair of resident alleles $\left(x_{1}, x_{2}\right)$ inside the area of protected polymorphisms, Eq. (3) determines whether a mutant of $x_{1}$ or $x_{2}$ can invade. Like in the monomorphic case, the alleles undergo directional coevolution inside most parts of the area of protected polymorphisms. Lines inside the area of protected polymorphisms at which directional evolution of $x_{1}\left(x_{2}\right)$ ceases are called $x_{1}-\left(x_{2}-\right)$ isoclines (Fig. 2, left panels; Geritz et al., 1998). The points of an isocline are analogous to monomorphic singularities: For example, if $x_{2}$ were fixed and only $x_{1}$ could mutate, then on the isocline $x_{1}$ may be evolutionarily stable or may undergo branching. The intersections of isoclines are the polymorphic evolutionary singularities where none of the two alleles undergo directional evolution. Such a singularity is an evolutionarily stable polymorphism if both isoclines are evolutionarily stable at their intersection. For convergence stability in polymorphic populations see Matessi and Di Pascuale (1996).

The evolution of a polymorphic population can be envisaged as a stochastic broken-line trajectory in the area of protected polymorphisms. When a larger (smaller) mutant of $x_{1}$ substitutes the original resident allele, the population moves horizontally to the right (left); substitution of $x_{2}$ by a larger (smaller) mutant corresponds to a vertical step upwards (downwards). The length of each step is random within some small mutation radius. Since only a finite number of substitutional steps is needed to reach the close neighbourhood of a convergence stable singularity, the evolutionary trajectory is subject to sampling stochasticity.

The left panels of Fig. 2 show the isoclines derived from Eq. 3 for three values of $d / \sigma$, assuming equal patch sizes $\left(c_{1}=c_{2}=0.5\right)$. The symmetry of the isocline plots in the main diagonal $(y=x)$ is due to the arbitrary order of labeling the resident alleles. Symmetry in the second diagonal $(y=-x)$ is a consequence of the patches being of equal size. 
If the difference between the optimal phenotypes in the first and in the second patch is moderate (Fig. $2 \mathrm{a} ; d / \sigma=2.25$ ), there is a single convergence stable and evolutionarily stable polymorphism of two alleles $(-0.81,0.81)$. The alleles are arranged symmetrically on either side of the branching point $\left(x^{*}=0\right)$. If the difference between the optimal phenotypes is greater, then the isoclines intersect three times (Fig. $2 \mathrm{~b} ; d / \sigma=3$ ): There are two convergence stable and evolutionarily stable singularities at $(-1.43,4.35)$ and $(-4.35,1.43)$, separated by an evolutionary saddle at $(-1.46,1.46)$. Though the model is symmetric in terms of relative patch size $\left(c_{1}=c_{2}=0.5\right)$, the alleles of a convergence stable singularity are now asymmetric relative to the branching point $\left(x^{*}=0\right)$. The population may evolve to either convergence stable singularity, depending on the sampling stochasticity of the broken-line trajectories. If the difference between the optimal phenotypes is very large (Fig. 2c; $d / \sigma=5)$, then three convergence stable and evolutionarily stable singularities exist, one symmetrical $(-2.5,2.5)$ and two asymmetrical $((-2.5$, $7.5)$ and $(-7.5,2.5))$.

The right panels of Fig. 2 show the corresponding simulated evolutionary trees (see Appendix A for the details of the simulations). The evolutionary trees confirm the predictions of evolutionary branching at $x^{*}=0$ and of reaching a final stop at one of the convergence stable and evolutionarily stable polymorphisms. In contrast to the assumptions used for the analysis of the model, in the simulations mutations occur rather frequently, and the population does not reach its population genetical equilibrium before a new mutant comes along. Due to recurrent mutations and incomplete allele substitution, there is always some polymorphism within the branches of the evolutionary tree. However, this apparently does not confound the predicted course of evolution. The predictions turn out to be fairly robust with respect to the size of mutations as well. 


\section{Bifurcation Analysis}

In the next part of the paper we perform a bifurcation analysis of monomorphic and polymorphic evolutionary singularities, in order to investigate the conditions under which evolutionary branching occurs, and the characteristics of the resulting evolutionarily stable polymorphisms.

\section{Monomorphic singularities}

The position of the monomorphic evolutionary singularity and its stability properties can be determined analytically (see Appendix C). There is always a single monomorphic singularity,

$$
x^{*}=c_{1} m_{1}+c_{2} m_{2}
$$

The singularity corresponds to a generalist phenotype, i.e., a type that is intermediate between the optimal phenotypes in the two patches. If the relative sizes of the patches are unequal, the singularity is nearer to the optimum of the larger patch.

The monomorphic singularity is always convergence stable, i.e., a monomorphic population first evolves to the generalist phenotype. However, the singularity is evolutionarily stable only if

$$
(d / \sigma)^{2}<\frac{1}{c_{1} c_{2}}
$$

If this condition is not satisfied, then the singularity is a branching point, giving rise to the evolution of a polymorphism. The difference between the two optimal phenotypes required for evolutionary branching is smallest if the patches are of equal size; the more asymmetric the relative size of the patches is, the greater difference between the optimal phenotypes is necessary in order to get evolutionary branching (Fig. 3). 


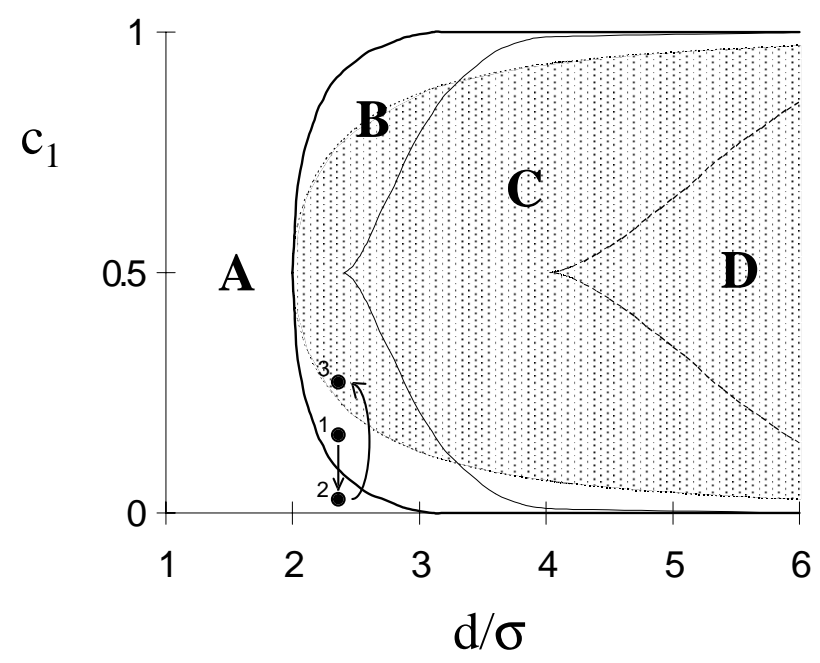

Fig. 3. Bifurcation plot of evolutionary singularities. Inside the dotted area, the monomorphic population has a branching point, outside this area the monomorphic population has an evolutionarily stable allele. The population has no polymorphic singularity in area A (left to the thick line), a single convergence stable as well as evolutionarily stable polymorphism in area B (between the thick line and the thin solid line), two convergence stable and evolutionarily stable polymorphisms separated by an evolutionary saddle in area C (between the thin solid line and the dashed line), and three convergence stable polymorphisms in area $\mathrm{D}$ (right to the dashed line). The numbered dots illustrate an evolutionary hysteresis effect (see the Discussion).

\section{Polymorphic singularities}

The number, position, and stability properties of polymorphic singularities were determined numerically. Fig. 3 shows the parameter regions with respectively no, one, two, or three convergence stable singularities; the position of the polymorphic evolutionary singularities as a function of relative patch size are shown in Fig. 4 for three values of $d / \sigma$. Except for a very narrow parameter range (see below), all polymorphic convergence stable singularities with two alleles are evolutionarily stable as well, i.e., there is no further branching to polymorphisms of more than two alleles. Notice that evolutionarily stable polymorphisms may exist even if the monomorphic population has an ESS: Such polymorphisms cannot be reached by an initially monomorphic population, but could be maintained indefinitely if the population was polymorphic from the onset. 
(a)

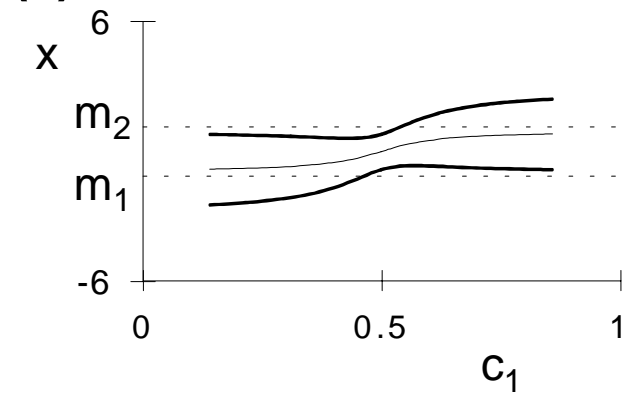

(b)
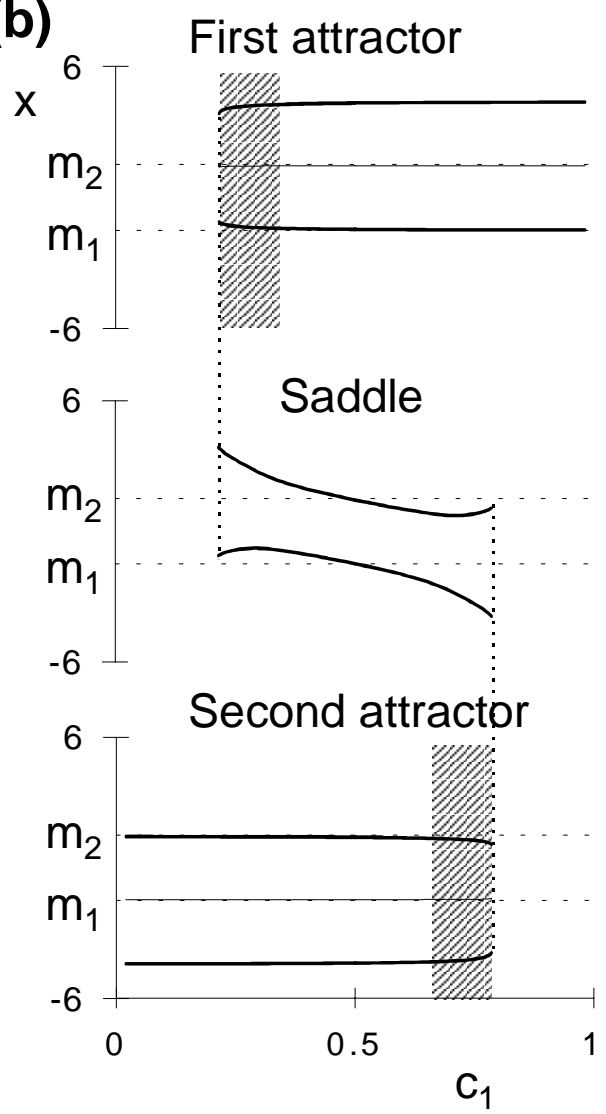

(c)
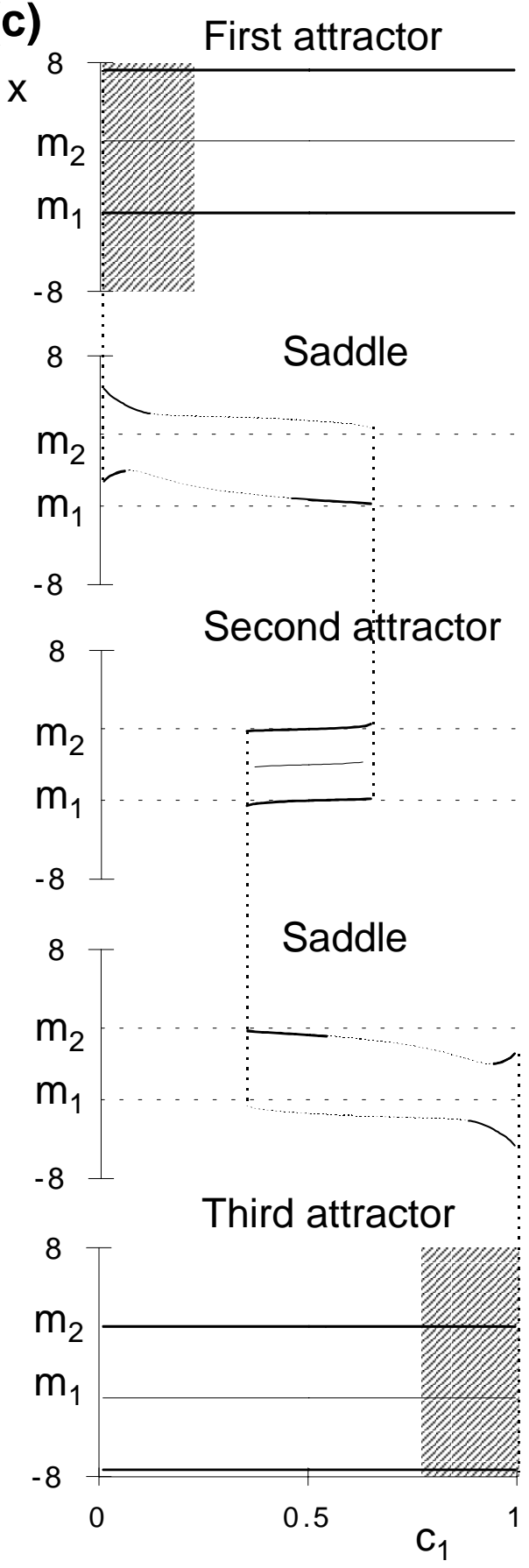

Fig. 4. The position of evolutionary singularities as a function of relative patch size. Thick lines represent the evolutionarily stable alleles or, equivalently, the homozygote phenotypes; thin dotted lines correspond to evolutionarily unstable singular alleles; thin solid lines show the phenotype of the heterozygote in the convergence stable polymorphisms. Different singularities are shown in separate plots, vertical dotted lines indicate fold bifurcations. In the shaded parts the singularity cannot be reached from the branching point. (a) $d / \sigma=2.25$; (b) $d / \sigma=3$; (c) $d / \sigma=5$. 
For moderate values of the difference between the optimal phenotypes $(2<d / \sigma<2.41)$, there is a single convergence stable polymorphism for all values of $c_{1}$ (Fig. 4a). If the two patches have approximately the same size $\left(c_{1} \approx 0.5\right)$, then the two homozygote phenotypes are near the within-patch optima, while the intermediate heterozygote is suboptimal in both patches. This arrangement is, however, not robust with respect to relative patch size. If the patches differ substantially in size, then one homozygote is almost optimal in the large patch, and the other homozygote has an extreme phenotype such that the intermediate heterozygote is almost optimal in the small patch. For lower values of $d / \sigma$, the 'almost optimal' phenotypes are closer to each other and differ more from $m_{1}$ and $m_{2}$. This is so because a slight difference from the optimum does not decrease the within-patch fitness noticeably (the fitness function is flat at its top), but it increases fitness in the other patch.

If the difference between the optimal phenotypes is greater $(2.41<d / \sigma<4.03)$, there are two convergence stable polymorphic singularities, the position of which remains approximately constant over a wide range of relative patch size (Fig. 4b). At the first convergence stable singularity, one homozygote is almost optimal in the first patch, and the heterozygote is almost optimal in the second patch; the roles are reversed at the second convergence stable singularity. These arrangements appear in the asymmetric evolutionary trees of the symmetric model in Fig. 2b. At sufficiently unequal patch sizes, however, only one polymorphic singularity exists, where a homozygote is almost optimal in the large patch and the heterozygote is almost optimal in the small patch.

For very large values of the difference between the optimal phenotypes $(d / \sigma>4.03)$ there are three convergence stable singularities (Fg. $4 \mathrm{c})$. Two of them (the first and the third ones in Fig. 4c) are similar to those which appear in Fig. $4 \mathrm{~b}$, except that they exist for virtually the whole range of relative patch size. At 
the remaining convergence stable singularity in the middle (the second one in Fig. 4c), the two homozygotes are almost optimal in the two patches, and the heterozygote has low fitness in both patches. This convergence stable singularity lacks evolutionary stability for two very narrow ranges of relative patch size $\left(0.348<c_{1}<0.352\right.$ and $0.648<c_{1}<0.652$ for $d / \sigma=5$, too narrow to be shown in Fig. $4 c)$, just before it disappears through a fold bifurcation; all other convergence stable polymorphic singularities are evolutionarily stable. Simulations of the evolutionary tree show that in these narrow parameter ranges, the population undergoes secondary branching at the polymorphic singularity resulting in three alleles. Soon after secondary branching, however, one allele goes extinct, and the population evolves to the first $\left(0.648<c_{1}<0.652\right)$ or to the third convergence stable singularity $\left(0.348<c_{1}<0.352\right)$.

\section{Evolution with multiple attractors}

If there exist several convergence stable polymorphic singularities, the probability of evolving to one or another can be markedly different. Recall that evolution in our model proceeds by finite steps along broken-line trajectories subject to sampling stochasticity, thus the course of evolution is not determined unequivocally. Consider first the case of two convergence stable singularities. If the patches have exactly the same size $\left(c_{1}=c_{2}=0.5\right)$, and the population enters the area of protected polymorphisms exactly at the branching point, then due to symmetry, the population evolves with equal probabilities to the first or to the second convergence stable singularity. However, if the population evolves to the branching point from a distance, then it becomes polymorphic slightly before reaching the branching point, i.e., as soon as a mutant is inside the area of protected polymorphisms. The convergence stable singularity that is nearer to the initial polymorphic population is reached with higher probability. 

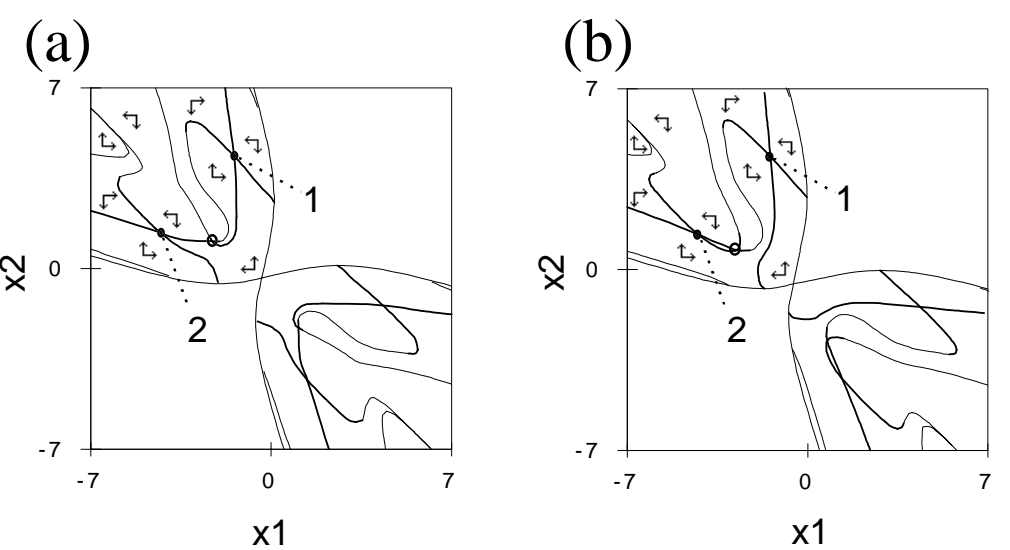

Fig. 5. Asymmetric isocline plots for $d / \sigma=3$ (notations as in Fig. 2). (a) $c_{1}=0.63$; $(b)$ $c_{1}=0.7$.

Unequal patch size also changes the probability of reaching one or the other convergence stable singularity. Moderate asymmetry in the relative patch size $\left(0.34<c_{1}<0.66\right.$ for $\left.d / \sigma=3\right)$ moves the saddle point into an asymmetric position relative to the branching point (compare Fig. 5a with the symmetric case shown in Fig. 2b). The population is more likely to evolve to the convergence stable singularity that is on the same side of the saddle where the branching point is. For example, the second convergence stable singularity can be reached from the branching point in Fig. $5 \mathrm{a}\left(c_{1}=0.63\right)$ only if, by chance, more (or larger) mutations occur in $x_{1}$ than in $x_{2}$. However, if mutations are small and many mutations are necessary to reach a singularity, such a sampling error has only low probability.

If the asymmetry of patch sizes is more pronounced $\left(0.22<c_{1}<0.34\right.$ or $0.66<c_{1}<0.79$ for $\left.d / \sigma=3\right)$, then there are still two singularities which are locally convergence stable, but one of them cannot be reached by a population that enters the area of protected polymorphism near the branching point. In Fig. 5b $\left(c_{1}=0.7\right)$, the evolutionary trajectory necessarily hits the $x_{1}$-isocline, and thereafter proceeds along this isocline upwards to the first convergence stable singularity. In Fig. 4, shaded parts mark the convergence stable singularities that are isolated from the branching point. 


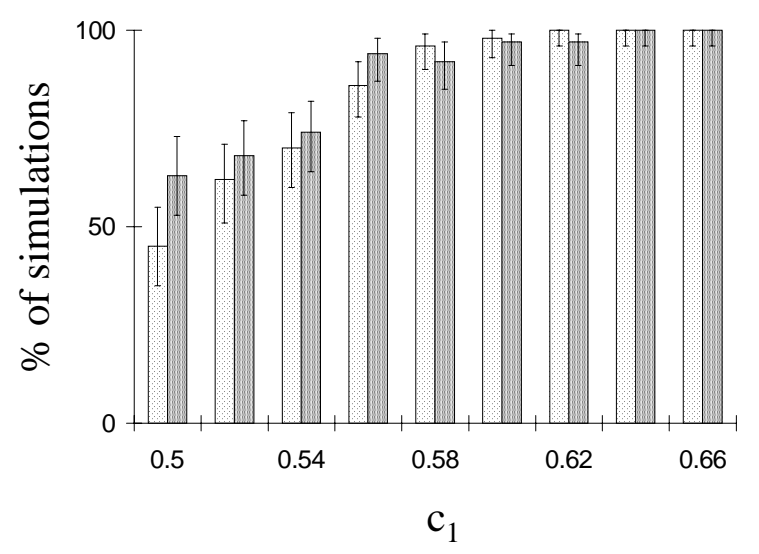

Fig. 6. Percentage of simulations evolving to singularity ' 1 ' as a function of relative patch size. Simulations starting below the branching point $\left(x_{i n i}=-1\right)$ are shown by the light columns, simulations starting above the branching point $\left(x_{i n i}=1\right)$ are represented by the dark columns. The error bars show the $95 \%$ confidence intervals of the measured percentages. $d / \sigma=3$, mutation stepsize $0.1,100$ simulations for each column. Notice that the scale of the horizontal axis runs only from 0.5 to 0.66 .

In order to assess the actual probabilities of arriving at the first versus at the second convergence stable singularity, we run a series of simulations starting with a monomorphic population either above or below the branching point for a range of relative patch sizes and $d / \sigma=3$ (Fig. 6). If the relative patch sizes are equal $\left(c_{1}=0.5\right)$, then simulations starting above the branching point reach the first singularity significantly more often than simulations starting below the branching point $\left(\chi^{2}=6.52\right.$, d.f. $\left.=1, P<0.02\right)$, because a population approaching the branching point from above (below) enters the area of protected polymorphisms slightly nearer to the first (second) singularity. If the first patch is larger than the second patch by just a few percent, the probability of evolving to the first convergence stable singularity markedly increases, till the second singularity becomes unreachable from the branching point (at $c_{1}=0.66$ in Fig. 6). The difference between simulations starting below and above the branching point quickly disappears as the size of the patches becomes unequal, such that the direction of evolution is governed by the asymmetry of the isocline plot. 
(a)

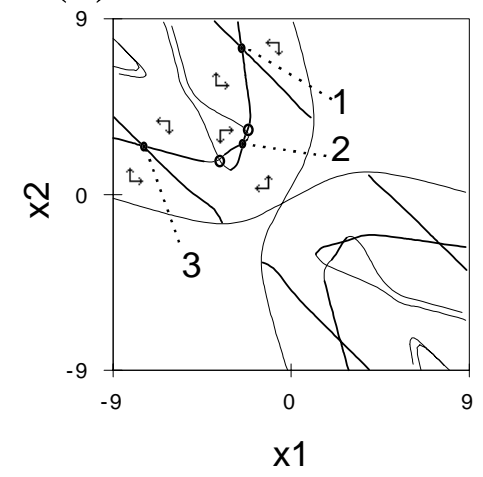

(b)

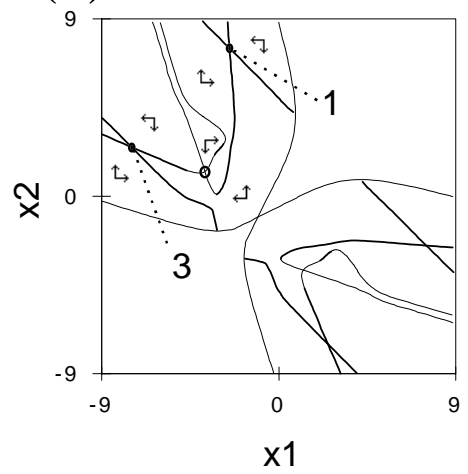

Fig. 7. Asymmetric isocline plots for $d / \sigma=5$ (notations as in Fig. 2). (a) $c_{1}=0.6$; (b) $c_{1}=0.75$.

Three convergence stable singularities occur only for very large differences between the optimal phenotypes in the first and in the second patch. Unfortunately, in this case the simulations require much larger computational capacity (see Appendix A), such that a statistical survey similar to the one shown in Fig. 6 was not feasible. If the patches are of equal size (Fig. 2c), the population entering the area of protected polymorphism at the branching point $\left(x^{*}=0\right)$ proceeds by small evolutionary steps most probably in the vicinity of the second diagonal $\left(x_{2}=-x_{1}\right)$, and therefore arrives at the middle convergence stable singularity (the second one in Fig. 2c). Since the two saddles direct the trajectories from the branching point towards the middle convergence stable singularity, the population will reach this singularity with highest probability even if the patch sizes are not equal (Fig. 7a). If the patch sizes are so different that the second singularity does not exist (Fig. 7b), then the population is more likely to stay on the side of the remaining saddle where the branching point is. Compared to Fig. $7 \mathrm{a}$, the probability of arriving at the first singularity thus markedly increases in Fig. 7b. In case of strongly different patch sizes one convergence stable singularity becomes isolated from the branching point (shaded parts in Fig. 4c). 


\section{Discussion}

\section{Evolution of genetic polymorphism in heterogeneous environments}

The maintenance of genetic polymorphism in heterogeneous environments under soft selection is a classical result of population genetics (Levene, 1953). In this paper, we investigated how such a polymorphism may arise in the first place, and how it will evolve, when mutations of only small phenotypic effect occur. To this end, we studied the adaptive dynamics of a diploid panmictic population in a two-patch environment with soft selection under the assumption of mutationlimited evolution.

We found that a monomorphic population first always evolves to a generalist phenotype. The generalist utilizes both patches, although in case of unequal patch sizes its phenotype is nearer to the optimal phenotype in the larger patch. If the difference between the optimal phenotypes in the two patches is small, then the generalist is evolutionarily stable. However, if the difference is large enough, then the population undergoes evolutionary branching, and gradually evolves to an evolutionarily stable genetic polymorphism with two distinct alleles. For a wide range of parameter values, one of the homozygotes and the heterozygote become specialists for the two patches, while the other homozygote has low fitness in both patches. Many models of multiple niche polymorphism assume that the two homozygotes have the highest fitness in the first and in the second patch, respectively. In the present model, however, such a polymorphism is reached by gradual evolution only in two separate parameter ranges, i.e., if the difference between the optimal phenotypes is moderate and the patches are not too different in size, or if the difference between the optimal phenotypes is very large. 
Several authors have pointed out that the maintenance of genetic polymorphism in heterogeneous environments is not robust when selection is weak: In order to maintain two alleles, the ratio of patch sizes must lie within a narrow range (Maynard Smith and Hoekstra, 1980; Hoekstra et al., 1985). Obviously, selection is weak if different alleles determine similar phenotypes. In our model, two similar alleles form a polymorphism at the beginning of evolutionary branching, nevertheless no fine-tuning of the relative patch size is necessary.

These seemingly conflicting results can be reconciled, however. For any given ratio of patch sizes, a polymorphism of similar alleles is possible only for some particular alleles within the continuous set of potential alleles. These particular alleles are in the neighbourhood of evolutionary singularities (Fig. 1). Since directional evolution leads towards a singularity, i.e., to those particular alleles which can be maintained by weak selection, protected polymorphism of similar alleles will develop. On an evolutionary timescale, polymorphism near an ESS is only temporary, and eventually the population will be monomorphic for the evolutionarily stable allele. Near a branching point, however, the polymorphism is preserved. As alleles diverge during evolutionary branching, the intensity of selection increases.

Strong selection is involved in the evolution of genetic polymorphism by evolutionary branching in two ways. First, within a patch, the ratio of the fitness of the optimal phenotype and the fitness of a far from optimal phenotype has to be sufficiently large in order to get branching (in case of Gaussian fitness functions, this condition is satisfied since phenotypes far from the optimum have vanishingly small fitness). Second, the difference between the optimal phenotypes in the two patches has to exceed a threshold. The minimal difference that is necessary for evolutionary branching depends on the shape of the fitness functions (Eqs. 1). Assuming that the within-patch fitness functions are symmetric and have identical shape, evolutionary branching is possible if the 
fitness functions are convex at their intersection (Appendix C). For Gaussian fitness functions, the minimal difference thus is $d / \sigma=2$ (cf. Fig. 3); the minimal difference would be smaller if the fitness functions were more peaked. Notice also that in Fig. 3, the range of the relative patch size that permits evolutionary branching expands fast with increasing the difference between the optimal phenotypes: Once $d / \sigma$ exceeds the threshold, the relative patch size is not very restrictive for evolutionary branching (compare with the wedge-shaped bifurcation diagrams of protected polymorphisms in Hoekstra et al., 1985).

Similarly to Levene (1953), we assumed that the number of adults recruited from each patch is constant ("soft selection"). Constant recruitment from each patch requires high fecundity, otherwise there may be not enough individuals left after selection to fill the patch. If the number of recruited adults is not constant, then a single specialist may be evolutionarily stable even if the unexploited patch is large (Meszéna et al., 1997). The reason for this is that if the population is specialized for one patch, then only a few individuals survive in the other patch. An allele slightly better adapted to the second patch and less to the first patch does not spread, because only a small fraction of adults is recruited from the patch where it is favoured. However, if the number of recruited adults is independent of the phenotype as in case of soft selection, then two specialists evolve via evolutionary branching. Soft selection thus facilitates the exploitation of different environments.

Evolutionarily stable genetic polymorphism can exist even if there is a monomorphic ESS for the same parameter values (Fig. 3). Although an initially monomorphic population cannot reach this polymorphism by small mutations, it may evolve if the initial population is polymorphic, or if a sufficiently different allele is introduced e.g. by occasional interspecific hybridization. The simultaneous existence of an ESS and an evolutionarily stable polymorphism 
produces an evolutionary hysteresis effect. Assume that the initial population is polymorphic (point 1 in Fig. 3), and that the relative patch size changes due to some change in the environment such that the polymorphism is lost (point 2 in Fig. 3). The population then evolves to the monomorphic ESS. Once the population is monomorphic, the polymorphism cannot be regained by simply restoring the original relative patch size, because the monomorphic population is still at an ESS. A substantially greater change in the environment is needed in order to make the evolution of polymorphism possible by small mutations (point 3 in Fig. 3). This phenomenon is not restricted to the present model, but appears to be a generic feature (Geritz et al., in prep.).

\section{Adaptive dynamics in diploid populations}

Comparison between the present model and its haploid counterpart (Geritz and Kisdi, in press) shows that the adaptive dynamics of a diploid population is much richer. In case of monomorphic populations the haploid and diploid models are similar; in particular, the conditions for evolutionary branching are identical. However, there are qualitative differences between haploid and diploid adaptive dynamics in polymorphic populations. After branching, the haploid population always evolves to a single evolutionarily stable coalition made up by two specialist phenotypes. In diploid populations, there can be up to three convergence stable polymorphisms, often with markedly different probabilities of reaching them.

A modelling approach related to ours was used by Christiansen and Loeschcke (1980) to study the evolution of resource exploitation. They assumed only a limited set of alleles covering a small range of phenotypes, and concluded that only the outermost alleles remain when the population is near a phenotype which we call branching point. However, they did not follow further the evolution of polymorphic populations. 
In the model analysis we assumed that mutations are small and occur infrequently; as shown by our simulations, however, the model is fairly robust with respect to both the size and rate of mutations. A crucial assumption in our approach is that of the finite mutational steps. If mutations were infinitesimally small, we would recover the differential equation-based adaptive dynamics models. The latter models, however, are unable to capture evolutionary branching (Geritz et al., 1998). The probabilistic nature of arriving at one or the other convergence stable polymorphism is also a consequence of finite mutations. Another essential assumption is that the phenotype of the heterozygote is intermediate between the two homozygotes. If the heterozygote exceeds both homozygotes, then the marginal fitness $W_{x}(y)$ is a non-differentiable function of $x$ and $y$, and the evolutionary singularities cannot be determined in the way done in Appendix C (Andrea Pugliese, pers. comm.). In case of complete dominance (e.g., an allele for larger phenotype is always dominant over an allele for smaller phenotype), the evolutionary singularities are identical to those of a haploid population.

The assumption of a single locus controlling a quantitative trait is but a first step in modelling adaptive dynamics in diploid populations. Although by no means common, a single locus may have major effect on a continuous trait. In the African finch Pyrenestes o. ostrinus, Smith (1993) found that the width of the lower mandible was effectively determined by a single locus with two alleles. The two alleles correspond to two peaks of the estimated fitness function, i.e., they appear to form an evolutionarily stable polymorphism.

Preliminary simulations of the present model with two unlinked loci resulted in evolutionary branching in both loci, producing four gamete types. After evolutionary branching, however, one allele went extinct and this locus remained monomorphic, unless the difference between the optimal phenotypes was very 
large. At the evolutionarily attractor there were two gamete types phenotypically equivalent to the two alleles of the convergence stable singularity in the one-locus model. If the difference between the optimal phenotypes was very large, then both loci remained polymorphic. But with an extremely large difference between the optimal phenotypes, such a strong linkage disequilibrium developed between the loci, that the population virtually contained only two gamete types, which again were phenotypically equivalent to the convergence stable singularity of the one-locus model. The final outcome of the two-locus simulations thus resembled the one-locus model in a wide range of parameters. It is not yet known to what extent the results of the two-locus simulations generalize to multilocus traits.

Notice that we assumed a more flexible type of genetic variation than the one used in most multilocus population genetical models. The latter models usually consider a given number of alleles (e.g., two) per locus, each allele with small phenotypic effect only. In our model, however, a continuum of potential alleles was assumed for each locus. Though the immediately available mutations were near the current resident alleles at any time, there was no limit on how far a locus may evolve by repeated allele substitutions. Thus the phenotypic effect of a single locus was not constrained a priori to be small.

It is biologically plausible that a single allele may not have an arbitrarily large effect on a continuous trait. When we sufficiently constrained the range of available alleles in the two-locus simulations, then both loci stayed polymorpic. Evolutionary branching thus gave rise to a genetic variation similar to the one assumed in multilocus genetic models.

Evolutionary branching in haploid populations could be regarded as morphological speciation. Evolutionary branching in allele space produces protected polymorphism, but not phenotypically distinct lineages. Heterozygotes and recombinants with intermediate phenotypes are selected against during 
evolutionary branching (see Udovic (1980) and Wilson and Turelli (1986) for heterozygote disadvantage in protected polymorphisms). However, random mating and recombination restore the intermediate types in each generation, and thus prevent the evolution of a bimodal phenotypic distribution. Phenotypically distinct lineages may evolve only if assortative mating develops, i.e., if the population undergoes speciation. Though the evolution of assortative mating is highly controversial, some recent theoretical studies (e.g., Diehl and Bush, 1989; de Meeûs et al., 1993; Doebeli, 1996a; Johnson et al., 1996; Doebeli and Dieckmann, in press) as well as empirical evidences (e.g., Coyne and Orr, 1989, 1997; Johannesson et al., 1995; Noor, 1995; Schluter and Nagel, 1995; Saetre et al., 1997; Galis and Metz, 1998; Nagel and Schluter, 1998; Rundle and Schluter, 1998) seem to support the possibility.

\section{References}

Abrams, P. A., AND H. MATSUdA. 1997. Fitness minimization and dynamic instability as a consequence of predator-prey coevolution. Evol. Ecol. 11:1-20

Abrams, P. A., H. MATSUdA, AND Y. HARADA. 1993a. Evolutionarily unstable fitness maxima and stable fitness minima of continuous traits. Evol. Ecol. 7:465-487.

Abrams, P. A., Y. Harada, AND H. Matsuda. 1993b. On the relationship between quantitative genetic and ESS models. Evolution 47:982-985.

BRown, J. S., AND N. B. PAVlOVIC. 1992. Evolution in heterogeneous environments: Effects of migration on habitat specialization. Evol. Ecol. 6:360-382.

Brown, J. S., AND T. L. VinCENT. 1987a. Coevolution as an evolutionary game. Evolution 41:66-79.

BRown, J. S., AND T. L. VinCENT. 1987b. A theory for the evolutionary game. Theor. Pop. Biol. 31:140-166.

BROWN, J. S., AND T. L. VINCENT. 1992. Organization of predator-prey communities as an evolutionary game. Evolution 46:1269-1283.

Charlesworth, B. 1990. Optimization models, quantitative genetics, and mutation. Evolution 44:520-538.

Christiansen, F. B. 1991. On conditions for evolutionary stability for a continuously varying character. Am. Nat. 138:37-50. 
Christiansen, F. B., AND V. LOESCHCKE. 1980. Evolution and intraspecific exploitative competition. I. One locus theory for small additive gene effects. Theor. Pop. Biol. 18:297-313. temporal and spatial structure. Theor. Pop. Biol. 39:63-99.

CoHen, D., And S. A. Levin. 1991. Dispersal in patchy environments: The effects of temporal and spatial structure. Theor. Pop. Biol. 39:63-99.

Coyne, J. A., AND H. A. ORR. 1989. Patterns of speciation in Drosophila. Evolution 43:362-381.

Coyne, J. A., AND H. A. ORR. 1997. "Patterns of speciation in Drosophila", revisited. Evolution 51:295-303.

DiECKMANN, U., AND R. LAW. 1996. The dynamical theory of coevolution: A derivation from stochastic ecological processes. J. Math. Biol. 34:579-612.

DIECKMANN, U., P. MARROW, AND R. LAW. 1995. Evolutionary cycling in predator-prey interactions: Population dynamics and the Red Queen. J. theor. Biol. 176:91-102.

DIEHL, S. R., AND G. L. BUSH. 1989. The role of habitat preference in adaptation and speciation. Pp. 345-365 in D. Otte, and J. A. Endler, eds. Speciation and its consequences. Sinauer Associates, Inc., Sunderland.

DoEbeli, M. 1996a. A quantitative genetic model for sympatric speciation. J. evol. Biol. 9:893-909.

Doebeli, M. 1996b. An explicit genetic model for ecological character displacement. Ecology 77:510-520.

DOEBELI, M., AND U. DiECKMANN. Evolutionary branching with multi-locus genetics. in U. Dieckmann, and J. A. J. Metz, eds. Advances in adaptive dynamics. Cambridge University Press. In press.

DoEBELI, M., AND G. D. RUXTON. 1997. Evolution of dispersal rates in metapopulation models: Branching and cyclic dynamics in phenotype space. Evolution 51:1730-1741.

ESHEL, I. 1983. Evolutionary and continuous stability. J. theor. Biol. 103:99-111.

Eshel, I., U. Motro, AND E. SANSONE. 1997. Continuous stability and evolutionary convergence. J. theor. Biol. 185:333-343.

Galis, F., And J. A. J. MEtz. 1998. Why are there so many cichlid species? TREE $13: 1-2$.

GERITZ, S. A. H., AND É. KISDI. Adaptive dynamics and evolutionary branching in mutation-limited evolution. in U. Dieckmann, and J. A. J. Metz, eds. Advances in adaptive dynamics. Cambridge University Press. In press.

GERITZ, S. A. H., É. KISDI, AND P. A. ABRAMS. Modeling evolutionary dynamics when there is more than one demographic attractor. In prep.

Geritz, S. A. H., É. KisDi, G. MeszénA, AND J. A. J. Metz. 1998. Evolutionarily singular strategies and the adaptive growth and branching of the evolutionary tree. Evol. Ecol. 12:35-57. 
Geritz, S. A. H., E. VAn DER Meijden, AND J. A. J. Metz. Evolutionary dynamics of seed size and seedling competitive ability. Theor. Pop. Biol. In press.

Geritz, S. A. H., J. A. J. Metz, É. KisDi, And G. Meszéna. 1997. Dynamics of adaptation and evolutionary branching. Phys. Rev. Letters 78:2024-2027.

Hoekstra, R. F., R. BiJlsma, And J. Dolman. 1985. Polymorphism from environmental heterogeneity: Models are only robust if the heterozygote is close in fitness to the favoured homozygote in each environment. Genet. Res. Camb. 45:299-314.

Hofbauer, J., AND K. Sigmund. 1990. Adaptive dynamics and evolutionary stability. Appl. Math. Lett. 3:75-79.

Iwasa, Y., A. Pomiankowski, And S. NeE. 1991. The evolution of costly mate preferences. II. The 'handicap' principle. Evolution 45:1431-1442.

JoHANNESSON, K., E. Rolan-AlvareZ, AND A. EKENDAHL. 1995. Incipient reproductive isolation between two sympatric morphs of the intertidal snail Lyttorina saxatilis. Evolution 49:1180-1190.

Johnson, P. A., F. C. Hoppenstaedt, J. J. Smith, And G. L. Bush. 1996. Conditions for sympatric speciation: A diploid model incorporating habitat fidelity and non-habitat assortative mating. Evol. Ecol. 10:187-205.

KISDI, É., AND G. MEsZÉNA. 1993. Density dependent life history evolution in fluctuating environments. Pp. 26-62 in J. Yoshimura and C. Clark, eds. Adaptation in a stochastic environment. Lecture Notes in Biomathematics Vol. 98, Springer Verlag, Berlin.

KISDI, É., AND G. MESZÉNA. 1995. Life histories with lottery competition in a stochastic environment: ESSs which do not prevail. Theor. Pop. Biol. 47:191-211.

LAw, R., P. Marrow, AND U. DiecKmann. 1997. On evolution under asymmetric competition. Evol. Ecol. 11:485-501.

LEVEne, H. 1953. Genetic equilibrium when more than one ecological niche is available. Am. Nat. 87:331-333.

Ludwig, D., AND S. A. LEvin. 1991. Evolutionary stability of plant communities and the maintenance of multiple dispersal types. Theor. Pop. Biol. 40:285-307.

Marrow, P., R. LAW, AND C. CANnings. 1992. The coevolution of predator-prey interactions: ESSs and Red Queen dynamics. Proc. R. Soc. Lond. B 250:133-141.

MARRow, P., U. DIECKMANN, AND R. LAW. 1996. Evolutionary dynamics of predator-prey systems: An ecological perspective. J. Math. Biol. 34:556-578.

MATESSI, C., AND C. Di PASQUALE. 1996. Long-term evolution of multilocus traits. J. Math. Biol. 34:613-653.

MATHIAS, A., AND É. KISDI. Evolutionary branching and coexistence of germination strategies. in U. Dieckmann, and J. A. J. Metz, eds. Advances in adaptive dynamics. Cambridge University Press. In press.

Matsuda, H. AND P. A. Abrams. 1994a. Timid consumers: Self-extinction due to adaptive change in foraging and anti-predator effort. Theor. Pop. Biol. 45:76-91. 
Matsuda H. AND P. A. AbRAms. 1994b. Runaway evolution to self-extinction under asymmetrical competition. Evolution 48:1764-1772.

MaYnaRd Smith, J. 1982. Evolution and the theory of games. Cambridge University Press, Cambridge.

MAYNARD SMITH, J. 1989. Evolutionary Genetics. Oxford University Press.

MaYnard Smith, J., AND R. F. HoEKSTRA. 1980. Polymorphism in a varied environment: How robust are the models? Genet. Res. Camb. 35:45-57.

De Meeûs, T., Y. Michalakis, F. Renaud, And I. Olivieri. 1993. Polymorphism in heterogeneous environments, evolution of habitat selection and sympatric speciation: Soft and hard selection models. Evol. Ecol. 7:175-198.

Meszéna, G., I. Czibula, And S. A. H. Geritz. 1997. Adaptive dynamics in a 2-patch environment: A toy model for allopatric and parapatric speciation. J. Biol. Syst. 5:265-284.

MeszénA, G., AND J. A. J. MetZ. The role of effective environmental dimensionality. in U. Dieckmann, and J. A. J. Metz, eds. Advances in adaptive dynamics. Cambridge University Press. In press.

Metz, J. A. J., S. A. H. Geritz, G. Meszéna, F. J. A. Jacobs, and J. S. van HEERWAARDEN. 1996. Adaptive dynamics, a geometrical study of the consequences of nearly faithful reproduction. Pp. 183-231 in S. J. van Strien, and S. M. Verduyn Lunel, eds. Stochastic and spatial structures of dynamical systems. North Holland, Amsterdam, The Netherlands.

Metz, J. A. J., R. M. Nisbet, S. A. H. Geritz. 1992. How should we define 'fitness' for general ecological scenarios? TREE 7:198-202.

NAGEL, L., AND D. SCHLUTER. 1998. Body size, natural selection, and speciation in sticklebacks. Evolution 52: 209-218.

Noor, M. A. 1995. Speciation driven by natural selection in Drosophila. Nature 375:674-675.

NowAK, M. 1990. An evolutionary stable strategy may be inaccessible. J. theor. Biol. 142:237-241.

Rand, D. A., H. B. Wilson, J. M. McGlade. 1994. Dynamics and evolution: Evolutionarily stable attractors, invasion exponents and phenotype dynamics. Phil. Trans. R. Soc. Lond. B 343:261-283.

RoughgaRDEN, J. 1979. Theory of population genetics and evolutionary ecology: An introduction. MacMillan, New York.

RundLe, H. D., AND D. SCHLUTER. 1998. Reinforcement of stickleback mate preferences: Sympatry breeds contempt. Evolution 52:200-208.

Saetre, G. P., T. Moum, S. Bures, M. Kral, M. Adamuan, And J. Moreno. 1997. A sexually selected character displacement in flycatchers reinforces premating isolation. Nature 387:589-592.

SCHLUTER, D., AND L. M. NAgel. 1995. Parallel speciation by natural selection. Am. Nat. 146:292-301. 
SMith, T. B. 1993. Disruptive selection and the genetic basis of bill size polymorphism in the African finch Pyrenestes. Nature 363:618-620.

TAPER, M. L., AND T. J. CASE. 1992. Models of character displacement and the theoretical robustness of taxon cycles. Evolution 46:317-333.

TAYLOR, P. D. 1989. Evolutionary stability in one-parameter models under weak selection. Theor. Pop. Biol. 36:125-143.

TAYLOR, P. D. 1996. The selection differential in quantitative genetics and ESS models. Evolution 50:2106-2110.

Van Tienderen, P. H., And G. De Jong. 1986. Sex ratio under the haystack model: Polymorphism may occur. J. theor. Biol. 122:69-81.

Udovic, D. 1980. Frequency-dependent selection, disruptive selection, and the evolution of reproductive isolation. Am. Nat. 116:621-641.

Vincent, T. L., Y. COHEN, AND J. S. BROWN. 1993. Evolution via strategy dynamics. Theor. Pop. Biol. 44:149-176.

Wilson, D. S., AND M. Turelli. 1986. Stable underdominance and the evolutionary invasion of empty niches. Am. Nat. 127:835-850. 


\section{Appendix A}

\section{Simulated evolutionary trees}

The simulation algorithm consisted of two modules, a deterministic iteration of allele frequencies for sufficiently common allele types, and an individual-based simulation to account for demographic stochasticity when an allele type is rare. The latter module was included since the chance of loss of new mutant alleles by demographic stochasticity influences the speed of evolution, and the relative speed of evolution, in turn, determines the shape of the evolutionary trajectory in polymorphic populations. The shape of the trajectories is important for the results presented in Fig. 6.

The deterministic iteration was based on the equation

$$
p_{i}^{\prime}=p_{i}\left[c_{l} \frac{\sum_{j} p_{j} f_{l}\left(\frac{x_{i}+x_{j}}{2}\right)}{\sum_{j} \sum_{k} p_{j} p_{k} f_{l}\left(\frac{x_{j}+x_{k}}{2}\right)}+c_{2} \frac{\sum_{j} p_{j} f_{2}\left(\frac{x_{i}+x_{j}}{2}\right)}{\sum_{j} \sum_{k} p_{j} p_{k} f_{2}\left(\frac{x_{j}+x_{k}}{2}\right)}\right]
$$

where $p_{i}$ and $p_{i}^{\prime}$ are the frequencies of allele type $x_{i}$ in the present and in the next generation, respectively.

In the individual-based module, each individual allele was paired with an allele chosen randomly from the deterministic part (hence only heterozygotes with a single rare allele were considered), and assigned randomly to patch 1 (patch 2) with probability $c_{1}\left(c_{2}\right)$. Whether or not the heterozygote survived till reproduction was decided randomly, the probability being determined by its phenotype during the period of selection, and by the deterministic population during the period of competition. The number of offspring was binomially distributed, males did not differ from females in their number of offspring. 
Fecundity had to be sufficiently high such that enough offspring survive the period of selection to recruit the fixed number of adults from each patch: The expected number of offspring was 45 (with variance 4.5) for $d / \sigma \leq 3$ (Figs. 2a,b, and 6) but the expected number had to be 4500 (with variance 450) for $d / \sigma=5$ (Fig. 2c). Each offspring inherited the rare allele at random with probability 0.5. (The probability of assignment to patch 1 or 2 and fecundity cancel out in the deterministic dynamics, but influence demographic stochasticity.)

The dynamics of an allele type was modelled by the individual-based module as long as it was present in less than $N_{T}$ zygotes. Because of potentially high mortality rates, the threshold had to be set at high values, $N_{T}=1000$ for $d / \sigma \leq 3$, and $N_{T}=10^{5}$ for $d / \sigma=5$; unfortunately, this greatly increased the computational capacity needed for the simulations. When the number of alleles exceeded $N_{T}$, the allele type was introduced into the deterministic iteration at a frequency $N_{T} / N_{\text {total }}=0.01$ (implying a total population size of $10^{5}$ for $N_{T}=1000$ and that of $10^{7}$ for $N_{T}=10^{5}$ ). Conversely, if the frequency of an allele type dropped below 0.01 in the deterministic module, the allele type was moved into the individual-based module. An allele type was extinct if its number hit zero in the individual-based simulation.

New alleles were introduced by mutations. Each allele in the deterministic module mutated with a probability $10^{-5}$ per generation, such that the total number of mutations was Poisson-distributed. The mutants differred from the original allele by a small mutation stepsize $\delta=0.1$ in random direction. The evolutionary trees of Fig. 2 show the allele types present in the deterministic part (i.e., with a frequency greater than 0.01). 


\section{Appendix B}

\section{Unique equilibrium allele frequencies in two-allele polymorphisms}

In this appendix, we prove that under the present model assumptions, a polymorphic population with two alleles always has a unique stable population genetical equilibrium. In order to do so, first we derive a sufficient condition under which the Levene model has at most one polymorphic equilibrium; then we show that this condition is fulfilled whenever the alleles determine a trait additively, and the within-patch fitness is a Gaussian function of this trait.

Consider two alleles, $A_{1}$ and $A_{2}$, with frequencies $p$ and $q=1-p$, respectively, and denote the fitness of genotype $A_{j} A_{k}$ in patch $i$ by $U_{j k}^{(i)}$. The frequency of allele $A_{1}$ changes according to

$$
\begin{aligned}
\Delta p & =c_{1} \frac{p q}{2 \bar{U}^{(l)}} \frac{d \bar{U}^{(l)}}{d p}+c_{2} \frac{p q}{2 \bar{U}^{(2)}} \frac{d \bar{U}^{(2)}}{d p} \\
& =\frac{p q}{2} \frac{d}{d p}\left[c_{1} \ln \bar{U}^{(l)}+c_{2} \ln \bar{U}^{(2)}\right]
\end{aligned}
$$

where $\bar{U}^{(i)}=p^{2} U_{11}^{(i)}+2 p q U_{12}^{(i)}+q^{2} U_{22}^{(i)}$ is the average fitness within patch $i$. At a polymorphic equilibrium, $c_{1} \ln \bar{U}^{(l)}+c_{2} \ln \bar{U}^{(2)}$ must have an extremum. If both $\ln \bar{U}^{(l)}$ and $\ln \bar{U}^{(2)}$ are concave at any $p$, then $c_{1} \ln \bar{U}^{(l)}+c_{2} \ln \bar{U}^{(2)}$ has at most one extremum, so that there can be at most one polymorphic equilibrium.

The details of the following derivations have been performed using Mathematica 2.2.3. The second derivative $d^{2} \ln \bar{U}^{(i)} / d p^{2}$ is of the form $A / B^{2}$. $A$ is a mountain parabola as a function of $p$, with a maximal value of $U_{11}^{(i)} U_{22}^{(i)}-\left[U_{12}^{(i)}\right]^{2} . A$ is therefore negative for any $p$ if $U_{11}^{(i)} U_{22}^{(i)}-\left[U_{12}^{(i)}\right]^{2}<0$, in which case $\ln \bar{U}^{(i)}$ will be concave. This proves the following 
Lemma: If the within-patch genotypic fitnesses of the Levene model satisfy the conditions $U_{11}^{(1)} U_{22}^{(l)} /\left[U_{12}^{(1)}\right]^{2}<1$ and $U_{11}^{(2)} U_{22}^{(2)} /\left[U_{12}^{(2)}\right]^{2}<1$, then there is at most one polymorphic equilibrium of the two alleles.

Substituting

$$
\begin{aligned}
U_{11}^{(i)} & =\alpha_{i} \exp \left(-\frac{\left(x_{1}-m_{i}\right)^{2}}{2 \sigma^{2}}\right) \\
U_{12}^{(i)} & =\alpha_{i} \exp \left(-\frac{\left(\frac{x+x_{2}}{2}-m_{i}\right)^{2}}{2 \sigma^{2}}\right) \\
U_{22}^{(i)} & =\alpha_{i} \exp \left(-\frac{\left(x_{2}-m_{i}\right)^{2}}{2 \sigma^{2}}\right)
\end{aligned}
$$

yields $U_{11}^{(i)} U_{22}^{(i)} /\left[U_{12}^{(1)}\right]^{2}=\exp \left(-\frac{\left(x_{x}-x_{2}\right)^{2}}{4 \sigma^{2}}\right)$, which is smaller than 1 whenever $x_{1} \neq x_{2}$. It thus follows that in the present model, there is at most one polymorphic equilibrium. In the area of protected polymorphism, where both trivial equilibria $(p=0$ and $p=1)$ are unstable, there is a unique stable equilibrium allele frequency; moreover, if one trivial equilibrium is stable and the other is unstable, then there cannot be any polymorphic equilibrium, i.e., nonprotected polymorphisms of two alleles are not possible.

Notice that this proof does not exclude nonprotected polymorphisms of three alleles, and therefore does not guarantee that during directional evolution in a polymorphic population with two resident alleles, invasion of a third allele leads indeed to the substitution of one resident allele. However, we can show that if two alleles of the three are sufficiently similar (i.e., produced by a small mutation), then generically three alleles may be present in equilibrium only near an isocline (Kisdi, unpubl.). 


\section{Appendix C}

\section{Monomorphic singularities}

In order to determine the monomorphic singularities, recall that if the population is monomorphic for the singular allele $x^{*}$, then the marginal fitness $W_{x^{*}}(y)$ of small mutants must either be smaller than 1 for all $y \neq x^{*}$ (ESS) or greater than 1 for all $y \neq x^{*}$ (branching point) while $W_{x^{*}}\left(x^{*}\right)=1$ (Fig. 1). At the monomorphic singularity $W_{x^{*}}(y)$ thus has an extremum, and $x^{*}$ is implicitely determined by

$$
\left.\frac{\partial W_{x}(y)}{\partial y}\right|_{y=x=x^{*}}=\frac{1}{2}\left[c_{1} \frac{f_{1}^{\prime}\left(x^{*}\right)}{f_{1}\left(x^{*}\right)}+c_{2} \frac{f_{2}^{\prime}\left(x^{*}\right)}{f_{2}\left(x^{*}\right)}\right]=0
$$

Substituting the Gaussian fitness functions from Eqs. 1, the above equation can easily be solved in order to obtain Eq. 4.

The monomorphic singularity is convergence stable if and only if

$$
\left[\frac{\partial^{2} W_{x}(y)}{\partial x \partial y}+\frac{\partial^{2} W_{x}(y)}{\partial y^{2}}\right]_{y=x=x^{*}}=\frac{1}{2}\left[c_{1} \frac{f_{1}^{\prime \prime}}{f_{1}}+c_{2} \frac{f_{2}^{\prime \prime}}{f_{2}}-c_{1}\left(\frac{f_{1}^{\prime}}{f_{1}}\right)^{2}-c_{2}\left(\frac{f_{2}^{\prime}}{f_{2}}\right)^{2}\right]_{x^{*}}<0(\mathrm{C} 2)
$$

(Eshel, 1983). Notice that this condition is fulfilled if the fitness functions are concave or moderately convex at $x *$. For Gaussian fitness functions, this condition is equivalent to $1 / \sigma^{2}>0$, which is always satisfied.

The monomorphic singularity is an ESS if and only if

$$
\left.\frac{\partial^{2} W_{x}(y)}{\partial y^{2}}\right|_{y=x=x^{*}}=\frac{1}{4}\left[c_{1} \frac{f_{1}^{\prime \prime}}{f_{1}}+c_{2} \frac{f_{2}^{\prime \prime}}{f_{2}}\right]_{x^{*}}<0
$$

(Maynard Smith, 1982). The monomorphic singularity is evolutionarily unstable, and hence evolutionary branching is possible, if the fitness functions are convex 
at $x^{*}$; strong convexity, however, makes the singularity convergence unstable (see above). When the Gaussian fitness functions are substituted, and $x^{*}$ is replaced from Eq. 4, this condition reduces to inequality (5).

In order to get some insight into the bifurcation patterns, assume that $f_{1}$ and $f_{2}$ have identical shape (i.e., $\left.f_{2}(x)=f_{1}(x-d)\right)$, they are symmetric $\left(f_{1}\left(m_{1}+x\right)=f_{1}\left(m_{1}-x\right)\right)$, and analytical. Since $f_{1}$ and $f_{2}$ must be concave near their maxima, the singularity is an ESS if $d$ is sufficiently small. By symmetry, if evolutionary branching is possible with a certain value of $d$ and $c_{1}=c$, then it is also possible with $c_{1}=1-c$; it follows that branching may occur with the smallest between-patch difference if $c_{1}=0.5$. With $c_{1}=0.5$, the singular point coincides with the point where $f_{1}$ and $f_{2}$ intersect, and branching becomes possible when $f_{1}$ and $f_{2}$ are sufficiently apart such that they are convex at their intersection. Convergence stability may be lost at large values of $d$, if the fitness function is too convex far from its maximum; but this is not the case with Gaussian fitness functions. 